\title{
Circulating miR-21, miR-378, and miR-940 increase in response to an acute exhaustive exercise in chronic heart failure patients
}

\author{
Tianzhao Xu ${ }^{1,2, *}$, Qiulian Zhou ${ }^{1,2, *}$, Lin Che $^{3, *}$, Saumya Das ${ }^{4}$, Lemin Wang ${ }^{3}$, \\ Jinfa Jiang ${ }^{3}$, Guanghe $\mathrm{Li}^{3}$, Jiahong $\mathrm{Xu}^{3}$, Jianhua $\mathrm{Yao}^{5}$, Hongbao Wang ${ }^{5}$, Yue Dai ${ }^{5}$, \\ Junjie Xiao ${ }^{1,2}$ \\ ${ }^{1}$ Regeneration and Ageing Lab, Experimental Center of Life Sciences, School of Life Science, Shanghai University, Shanghai \\ 200444, China \\ ${ }^{2}$ Shanghai Key Laboratory of Bio-Energy Crops, School of Life Science, Shanghai University, Shanghai 200444, China \\ ${ }^{3}$ Department of Cardiology, Tongji Hospital, Tongji University School of Medicine, Shanghai 200065, China \\ ${ }^{4}$ Cardiovascular Division of the Beth Israel Deaconess Medical Center and Harvard Medical School, Boston, MA 02215, USA \\ ${ }^{5}$ Department of Cardiology, Yangpu Hospital, Tongji University School of Medicine, Shanghai 200090, China \\ *These authors have contributed equally to this work
}

Correspondence to: Junjie Xiao, e-mail: junjiexiao@ shu.edu.cn

Lemin Wang, e-mail: wanglemin@163.com

Keywords: microRNA, heart failure, exercise

Received: July 04, $2015 \quad$ Accepted: January 05, 2016

Published: January 21, 2016

\section{ABSTRACT}

Congestive heart failure (CHF) is a major cause of hospitalizations, morbidity, and mortality in Western societies. In addition to optimal medical and device therapy, exercise training is an important adjunct treatment option for CHF patients. MicroRNAs (miRNAs, miRs) participate in a variety of physiological and pathological processes. Dynamic regulation of circulating miRNAs during exercise in healthy persons and athletes has recently been documented, however, the response of circulating miRNAs to exercise in CHF patients is undetermined. Twenty-eight CHF patients underwent a symptom-limited incremental cardiopulmonary exercise test on a bicycle ergometer using a standardized exercise protocol of revised Ramp10 programs at Shanghai Tongji Hospital. Blood samples were collected before and immediately after an acute exercise session. RNA was extracted from the serum and selected miRNAs were determined using quantitative polymerase chain reactions. Moreover, inflammatory and muscle damage markers were determined by enzyme linked immunosorbent assays. We found that serum miR-21, miR-378 and miR-940 levels were significantly up-regulated immediately following an acute exercise while the rest were not changed. In addition, no robust correlation was identified between changes of these miRNAs and exercise capacity, muscle damage or inflammation. In conclusion, serum miR-21, $\mathrm{miR}-378$, and $\mathbf{m i R}-940$ increase in response to an acute exhaustive exercise in CHF patients. Further studies are needed to clarify the potential use of circulating miRNAs as biomarkers of exercise adaptation in CHF patients, and if they have any use as prognostic markers of cardiovascular outcomes.

\section{INTRODUCTION}

Congestive heart failure (CHF), a growing epidemic, is a major cause of hospitalizations, morbidity, and mortality [1]. In Western societies, the prevalence of CHF in the general population is $1-2 \%$ and increases to $10 \%$ among those aged over 75 and
$20 \%$ among those aged over 80 [2]. The improvement in mortality from acute coronary syndromes due to more rapid revascularization has coincided with an increase in the late sequelae of myocardial infarction, namely CHF [1]. In addition, an increase in the age of the population has led to a marked increase in the incidence of CHF with preserved ejection fraction [1]. 
Together, the prevalence of CHF is increasing at a more rapid pace than any other cardiovascular diseases in the developed countries $[1,2]$. This is likely to contribute to increased mortality, morbidity and health-care expenditures over the next few decades $[1,2]$.

Exercise training is an effective adjunct nonpharmacological treatment option for CHF patients not only to improve exercise capacity and quality of life, but also to decrease major cardiovascular events, including mortality and morbidity [3-5]. Therefore, exercise training has been recommended as an important cornerstone of therapy for CHF patients by ACC, AHA, and the Heart Failure Society of America [1, 6]. However, the underling cellular and molecular mechanism for benefical effects of exercise is still unclear [7].

MicroRNAs (miRNAs, miRs) are 19-22 nucleotides non-coding RNAs that negatively regulate gene expression at the post-transcriptional level via mRNA degradation or translational inhibition [8-10]. As a single miRNA can regulate hundreds of genes directly or indirectly, miRNAs have been central players of gene regulation [11]. Therefore, miRNAs participate in a variety of physiological and pathological processes. Dysregulation of miRNAs contributes to many diseases including cardiovascular diseases and cancers [12-15]. Recently, miRNAs have been found to be present in a stable form in the circulation and have been found to be dynamically regulated in response to physiological and pathological processes [16-19]. Intriguingly, circulating miRNAs may regulate gene expression in the target cells and tissues as a novel mode of cell-cell communication [20]. Dynamic regulation of circulating miRNAs during exercise in healthy persons and athletes has recently been documented, however, the response of circulating miRNAs to exercise in $\mathrm{CHF}$ patients is undetermined [21-25]. Here we investigate how specific circulating miRNAs with well-established roles in major adaptive processes is linked to exercise training in CHF patients. Specifically, we determined the expression levels' changes of circulating miRNAs before and after an acute exhaustive exercise in CHF patients. We found that serum miR-21, miR-378, and miR-940 increased in response to an acute exhaustive exercise in CHF patients. However, no robust correlation was identified between changes of these miRNAs and exercise capacity, muscle damage or inflammation, indicating further studies using high-throughput circulating miRNAs screening techniques are highly needed to identify the potential use of circulating miRNAs as biomarkers of aerobic exercise capacity.

\section{RESULTS}

\section{Subject characteristics}

The cohort used in this study is the same as previously reported [26]. A total of 28 male HF participants were enrolled with a mean age of $59.07 \pm 1.79 \mathrm{ys}$, height of $171.54 \pm 1.05 \mathrm{~cm}$, and body mass of $75.80 \pm 1.43$. The clinical characteristics for these subjects were shown in Table 1 . The average EF was $47.68 \pm 2.58 \%$. The detailed echocardiographic parameters for these participants were indicated in Table 2.

\section{Circulating miR-21, miR-378, and miR-940 increase in response to an acute exhaustive exercise in CHF patients}

The cardiac or muscle specific/enriched miRNAs including miR-1, miR-133a, miR-133b, miR-499, miR-208a, miR-208b, miR-378, miR-486, and miR-940 were determined. In addition, other miRNAs involved in angiogenesis (miR-328, miR-126, miR-221), inflammation (miR-21, miR-146a, miR-155), and ischemia adaptation (miR-210, miR-21, miR-146a) were also investigated. The majority of cardiac or muscle specific/enriched miRNAs except for miR-378, miR-486, and miR-940 displayed extremely low expression level as anticipated based on previous studies [23, 27]. Levels of serum miR-21, miR-378 and miR-940 were significantly up-regulated immediately following an acute exercise (Figure 1A-1C). In contrast, miR-1, miR-133a, miR-133b, miR-499, miR-208a, miR-208b, miR-486, miR-328, miR-126, miR-221, miR-146a, miR-155 and miR-210 were not changed following an exhaustive exercise (Figure 1A-1C). As at present, huge difference exist in choosing endogenous controls, it is recommend to have multiple endogenous controls to assure a robust finding regardless of the way of standardization [27]. Interestingly, when the values were normalized to miR-16, a similar tendency of serum levels of miR-21, miR-378 and miR-940 after an acute exercise was also found (Figure 1D). Collectively, these data consistently indicate that circulating miR-21, miR-378, and miR-940 increase in response to an acute exhaustive exercise in CHF patients.

\section{Correlations between changes of miR-21, miR-378, and miR-940 and exercise capacity, muscle damage or inflammation}

An acute exercise in CHF patients increased the levels of CK, LDH, and NT-ProBNP while did not affect CK-MB, Tn-T and hs-CRP (Table 3). As that has been analyzed in athlete before and after a marathon run in a previous report [26], here we also correlated the increase of circulating miRNAs induced by exercise to makers of cardiac function and exercise capacity as well as indicators of muscle damage and inflammation. As a robust correlation would be consistently found no matter choose cel-miR-39 or miR-16 as endogenous controls, here we failed to report a robust correlation between changes of miR-21, miR-378, and miR-940 and cardiac function 
Table 1: Clinical characteristics of participants

\begin{tabular}{|l|c|}
\hline \multicolumn{1}{|c|}{ Clinical Parameters } & Mean \pm SEM \\
\hline Age (years) & $59.07 \pm 1.79$ \\
\hline Height $(\mathrm{cm})$ & $171.54 \pm 1.05$ \\
\hline Body mass $(\mathrm{kg})$ & $75.80 \pm 1.43$ \\
\hline BMI $\left(\mathrm{kg} / \mathrm{m}^{2}\right)$ & $25.89 \pm 0.51$ \\
\hline Heart rate (beats $/ \mathrm{min})$ & $71.89 \pm 2.54$ \\
\hline Work load (watt) & $40.48 \pm 2.95$ \\
\hline $\mathrm{VO}_{2}$ max $(\mathrm{ml} / \mathrm{min} / \mathrm{kg})$ & $16.84 \pm 0.77$ \\
\hline Systolic blood pressure $(\mathrm{mmHg})$ & $114.29 \pm 3.45$ \\
\hline Diastolic blood pressure $(\mathrm{mmHg})$ & $73.57 \pm 2.44$ \\
\hline
\end{tabular}

Table 2: General echocardiographic indexes

\begin{tabular}{|l|c|c|}
\hline \multicolumn{1}{c}{ Clinical Parameters } & Mean \pm SEM & Normal Values \\
\hline Left ventricular end diastolic diameter $(\mathrm{mm})$ & $56.79 \pm 1.77$ & $35-56$ \\
\hline Left ventricular end systolic diameter $(\mathrm{mm})$ & $42.07 \pm 1.99$ & $23-35$ \\
\hline Interventricular septal thickness IVS $(\mathrm{mm})$ & $9.86 \pm 0.26$ & $6-11$ \\
\hline Left ventricular posterior wall thickness $(\mathrm{mm})$ & $8.46 \pm 0.29$ & $6-11$ \\
\hline Left atrial Dimension $(\mathrm{mm})$ & $46.89 \pm 1.07$ & $19-40$ \\
\hline Pulmonary arterial systolic pressure $(\mathrm{mmHg})$ & $35.62 \pm 2.01$ & $18-30$ \\
\hline Pulmonary artery diameter $(\mathrm{mm})$ & $26.25 \pm 0.52$ & $19-27$ \\
\hline Ejection fraction (EF\%) & $47.68 \pm 2.58$ & $60-75$ \\
\hline Aortic root dimension $(\mathrm{mm})$ & $34.46 \pm 0.55$ & $20-37$ \\
\hline
\end{tabular}

(Figure 2), exercise capacity (Figures 3-5), muscle damage or inflammation (Table 4), indicating further studies using miRNA arrays or RNA-seq are highly needed.

\section{DISCUSSION}

This study was designed to investigate how specific circulating miRNAs are modulated by an acute exhaustive exercise in CHF patients, and if there are differences compared to exercise adaptation in healthy control subjects or athletes that have been previously published [21-28]. Here we reported that serum miR-21, miR-378, and miR-940 increased in response to an acute exhaustive exercise in CHF patients. We also showed an acute exercise in these CHF patients increased the levels of CK, $\mathrm{LDH}$, and NT-ProBNP. Interestingly, no robust correlation is identified between the increase of circulating miRNAs induced by exercise to makers of cardiac function and exercise capacity as well as indicators of muscle damage and inflammation, indicating further studies using high-throughput circulating miRNAs screening techniques are highly needed. Moreover, the potential biological function of these dysregulated miRNAs responsible for beneficial effects of exercise training in CHF patients warrants further studied.
Several circulating miRNAs have been identified to respond to acute exhaustive exercise in healthy persons or athletes [21]. Specificially, circulating miR-1, miR-133, miR-499, miR-208, miR-146a, miR-221, miR-222, miR-338-3p, miR-330-3p, miR-223, miR-139-5p, miR-143, miR-21 and miR-149* were increased while miR-486, miR-106a, miR-30b, miR-151-5p, let-7i, miR-652 and miR-151-3p were robustly decreased after an acute exercise $[21,25,28]$. However, all these studies were conducted in healthy individuals or athletes. To the best of our knowledge, the present study is the first to identify serum miRNAs that change in response to exercise in CHF patients. Here we reported that serum miR-21, miR-378, and miR-940 increased in response to an acute exhaustive exercise in CHF patients while other miRNAs determined in this study were not changed, indicating that participants, exercise type, duration, and intensity might affect levels of circulating miRNAs [22]. In addition, these dysregulated miRNAs reported here are different from those reported in healthy control subjects or athletes, indicating a distinct exercise adaptation in CHF patients. Moreover, as most cardiac or muscle specific/enriched miRNAs were not changed in the present study, there must be distinct mechanisms of circulating miRNAs response to exercise rather than a general response to tissue damage. 


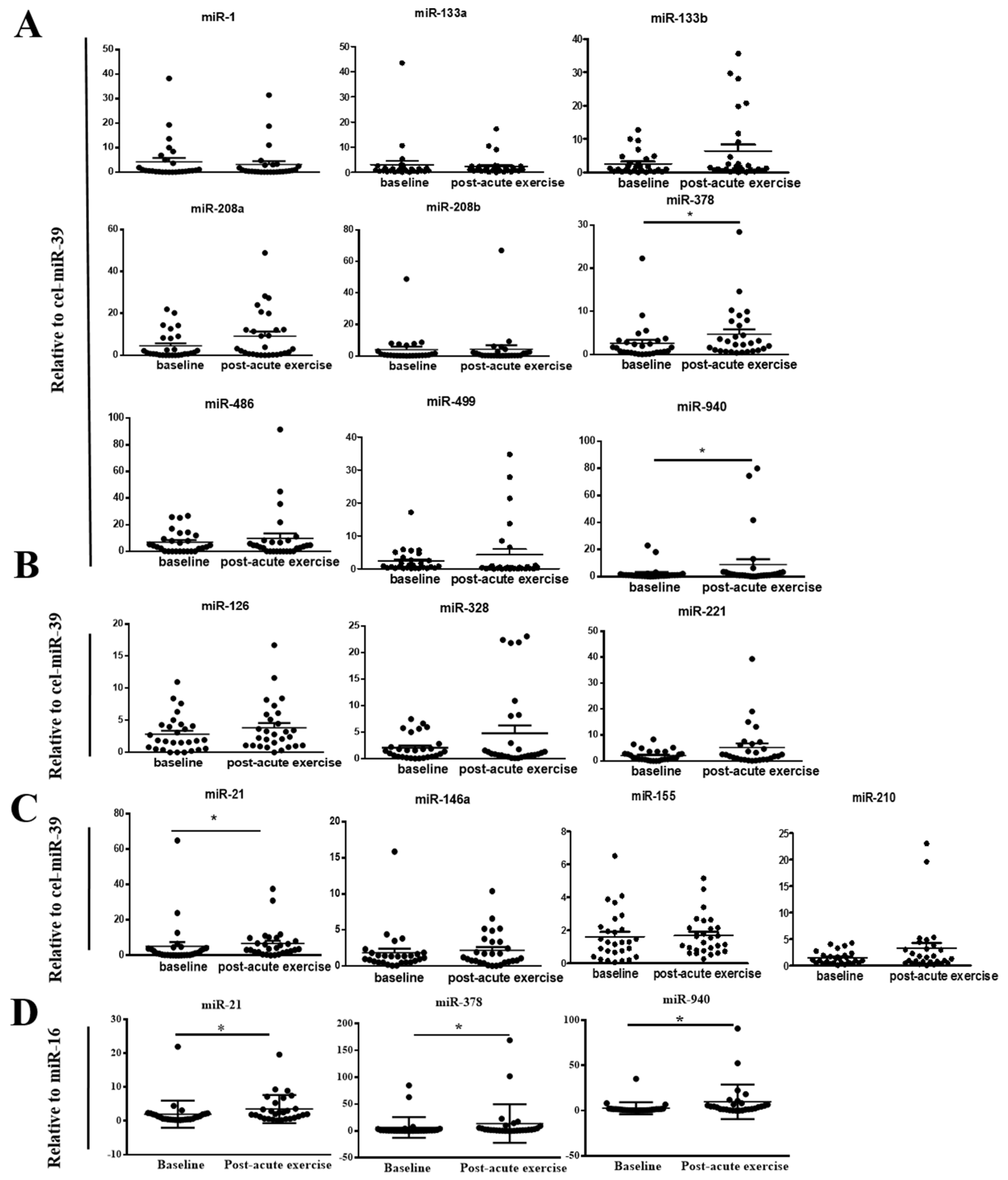

Figure 1: Changes in circulating microRNAs in response to an acute exhaustive exercise in chronic heart failure patients. (A) Changes of cardiac or muscle specific/enriched miRNAs (miR-1, miR-133a, miR-133b, miR-499, miR-208a, miR-208b, miR-378, miR-486, and miR-940) when normalized to cel-miR-39; *compared to the baseline, $P<0.05$. (B) Changes of miRNAs involved in angiogenesis (miR-328, miR-126, miR-221) when normalized to cel-miR-39; (C) Changes of miRNAs involved in ischemia adaptation and inflammation (miR-210, miR-21, miR-146a, miR-155) when normalized to cel-miR-39; *compared to the baseline, $P<0.05$; (D) Changes of miR-21, miR-378 and miR-940 when normalized to miR-16; *compared to the baseline, $P<0.05$. 
Table 3: Biochemical measurements

Baseline

After acute exercise

\begin{tabular}{|c|c|c|}
\hline \multicolumn{3}{|c|}{ Destruction parameters } \\
\hline $\mathrm{CK}(\mathrm{ng} / \mathrm{ml})$ & $6.48 \pm 1.12$ & $11.20 \pm 1.03^{*}$ \\
\hline LDH (U/l) & $2355.98 \pm 89.65$ & $2598.94 \pm 63.81^{*}$ \\
\hline \multicolumn{3}{|l|}{ Cardiac markers } \\
\hline CKMB (ng/ml) & $92.56 \pm 13.05$ & $80.60 \pm 11.32$ \\
\hline Troponin T (pg/ml) & $42.74 \pm 8.16$ & $44.23 \pm 8.60$ \\
\hline NT-ProBNP (pg/ml) & $193.38 \pm 32.94$ & $273.57 \pm 45.68^{*}$ \\
\hline \multicolumn{3}{|c|}{ Inflammatory marker } \\
\hline $\mathrm{Hs}-\mathrm{CRP}$ (ng/ml) & $9.845 \pm 1.22$ & $8.29 \pm 0.90$ \\
\hline
\end{tabular}

Values are means \pm SEM. CK, creatine kinase; LDH, lactate dehydrogenase; CK-MB, myocardial isoenzyme; Pro-BNP, pro-brain natriuretic peptide; hsCRP, high-sensitive C-reactive protein; *Significant difference between baseline/post-acute exercise.
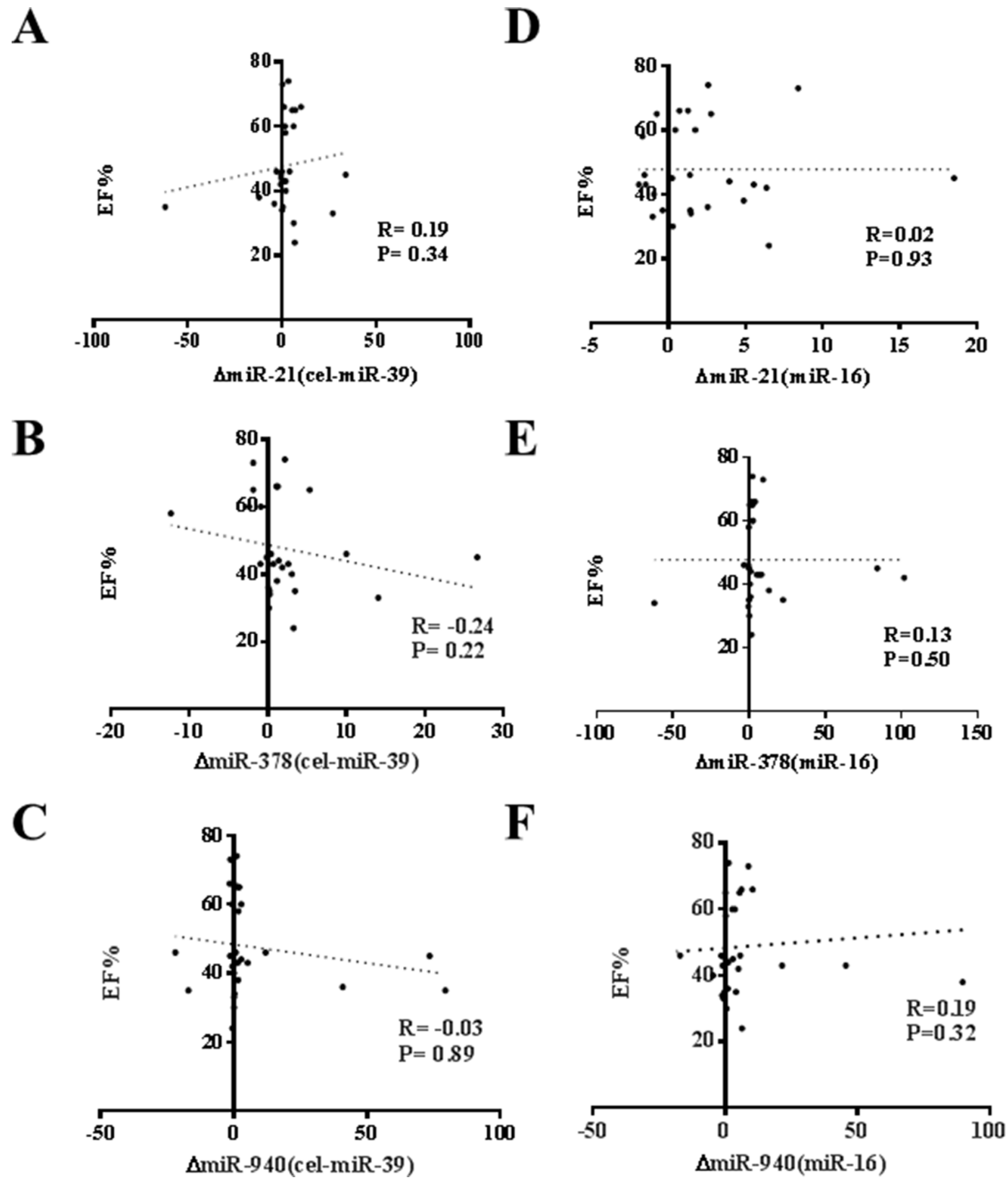

Figure 2: No correlation of changes of miR-21, miR-378 and miR-940 in exercise is observed with ejection fraction (EF\%). (A) miR-21 (normalized to cel-miR-39); (B) miR-378 (normalized to cel-miR-39); (C) miR-940 (normalized to cel-miR-39); (D) miR-21 (normalized to miR-16); (E) miR-378 (normalized to miR-16); (F) miR-940 (normalized to miR-16). 
A

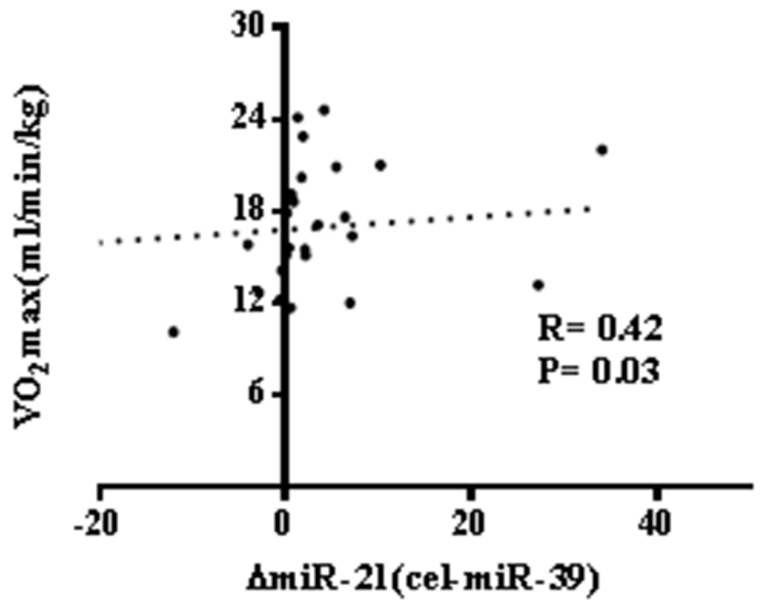

B

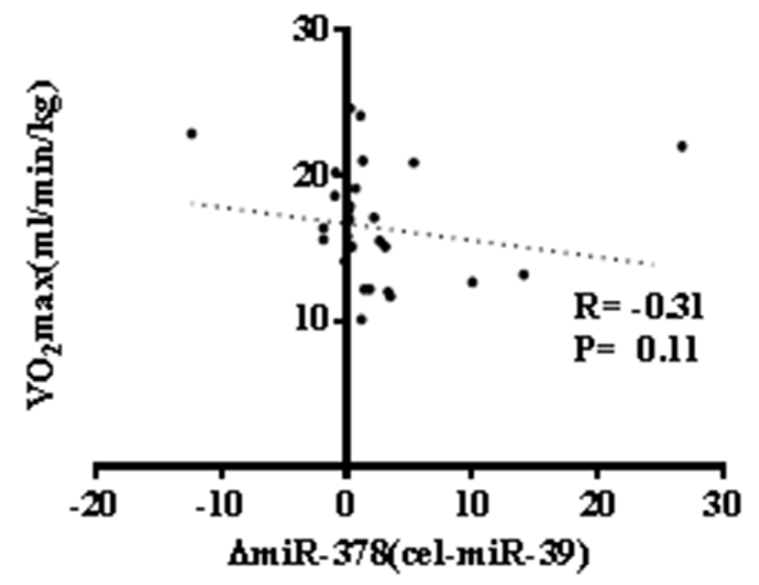

$\mathbf{C}$

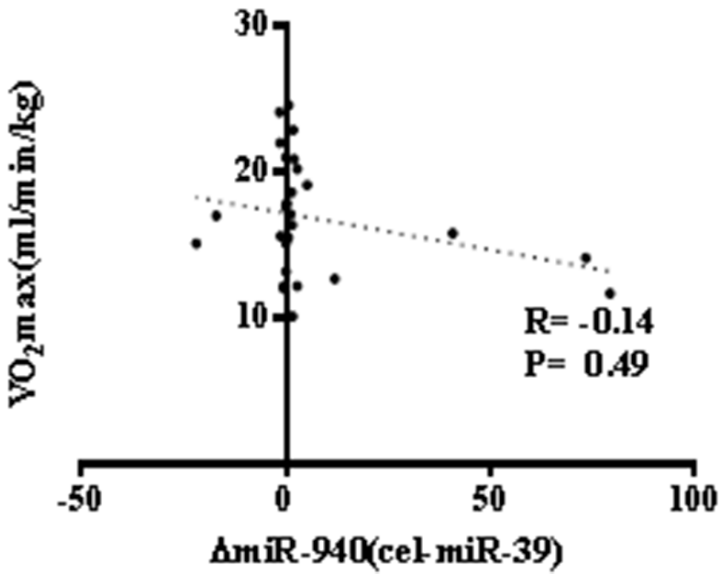

D

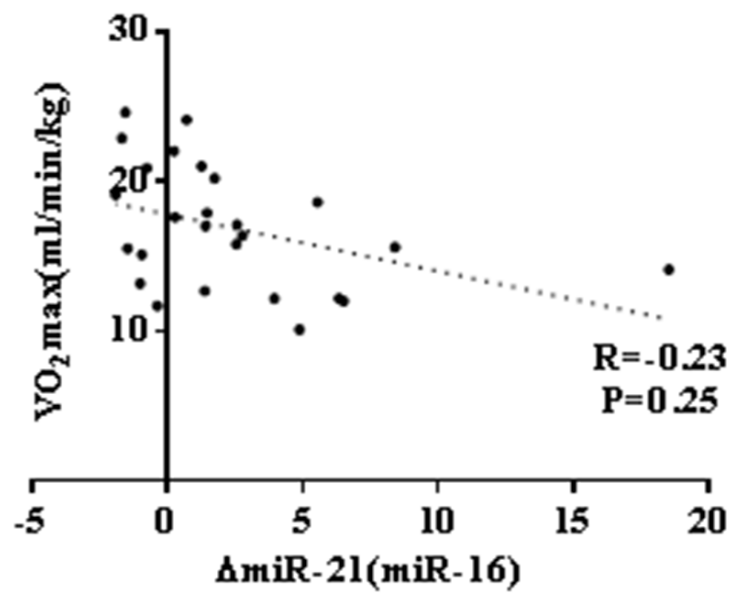

$\mathbf{E}$

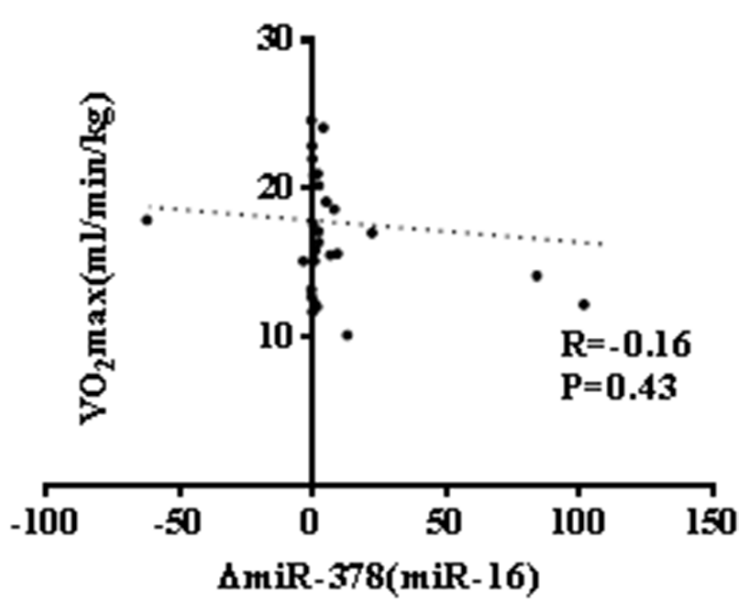

$\mathbf{F}$

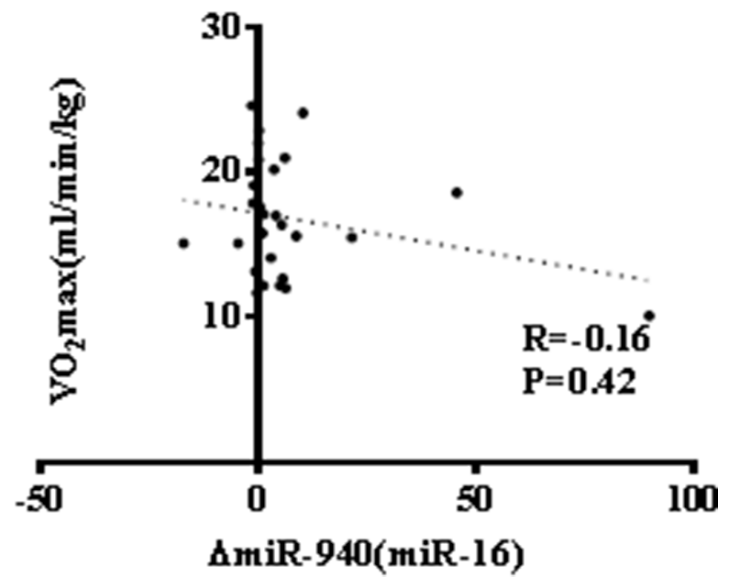

Figure 3: No robust correlation of changes of miR-21, miR-378 and miR-940 in exercise is observed with peak oxygen consumption $\left(\mathbf{V O}_{2 \max }\right)$. (A) miR-21 (normalized to cel-miR-39). Note: A moderate correlation of changes of miR-21 in exercise is observed with $\mathrm{VO}_{2 \max }$; (B) miR-378 (normalized to cel-miR-39); (C) miR-940 (normalized to cel-miR-39); (D) miR-21 (normalized to miR-16); (E) miR-378 (normalized to miR-16); (F) miR-940 (normalized to miR-16). 


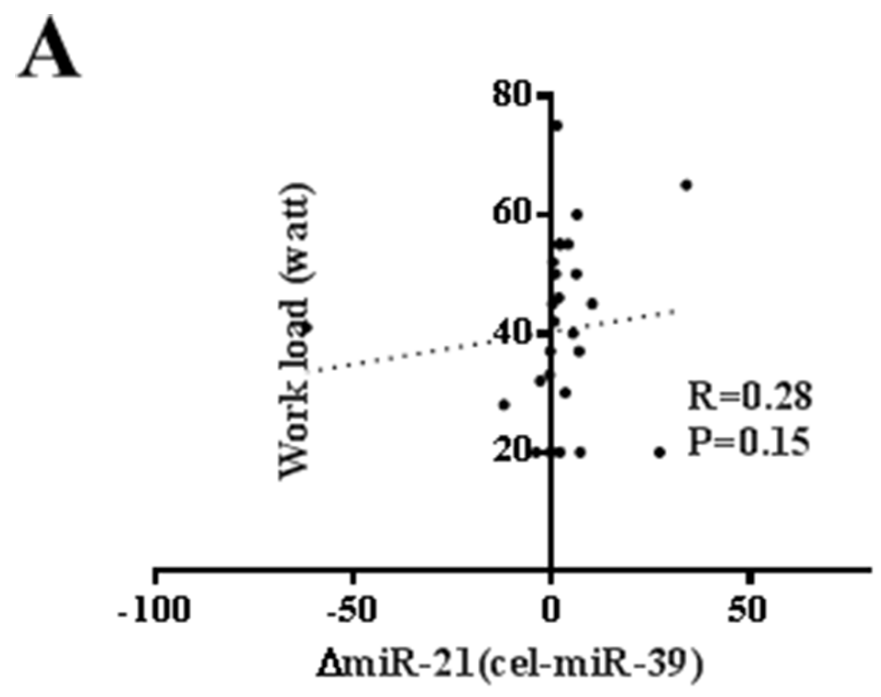

D

B

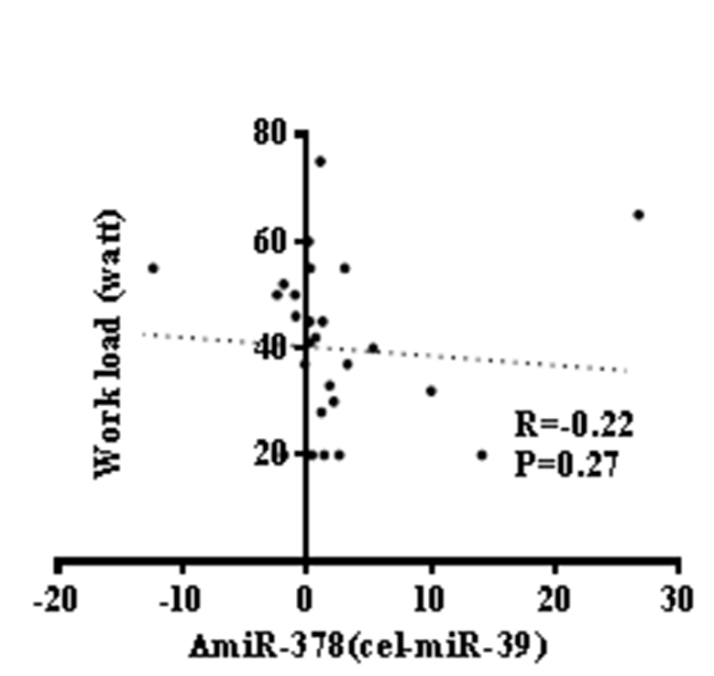

$\mathbf{E}$
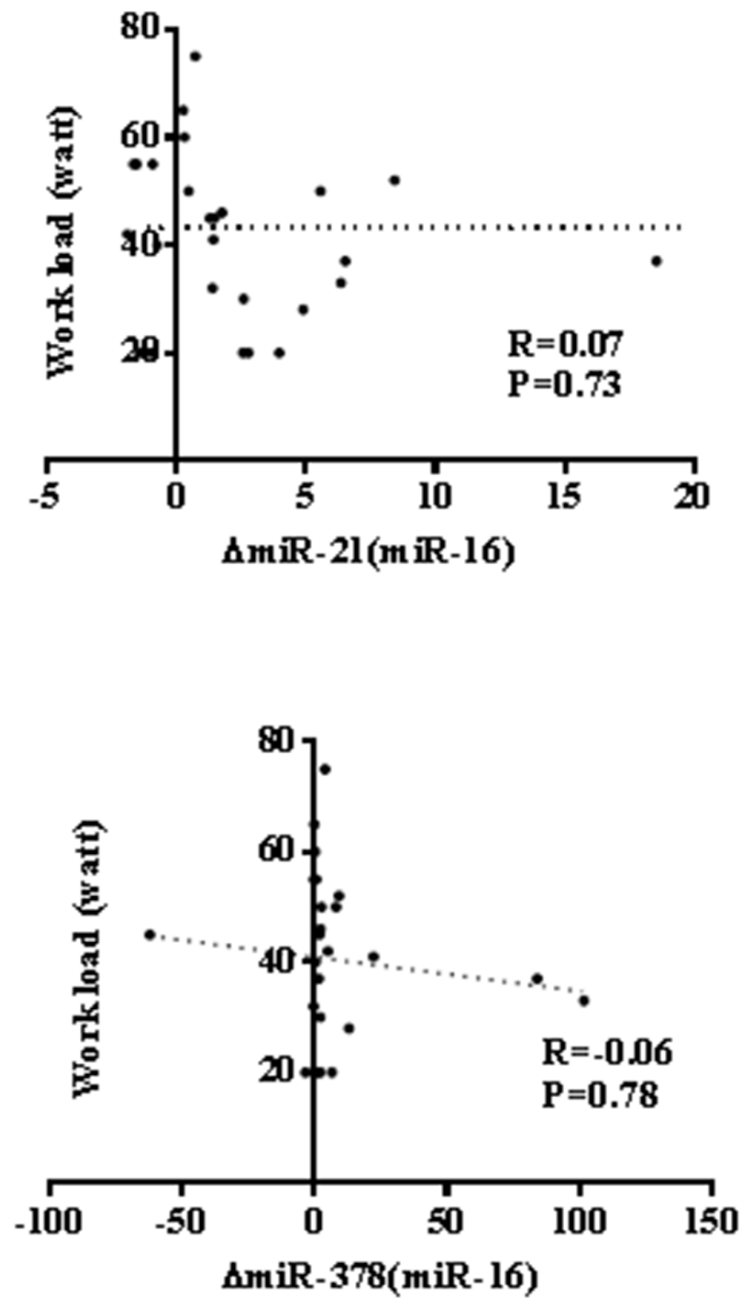

C
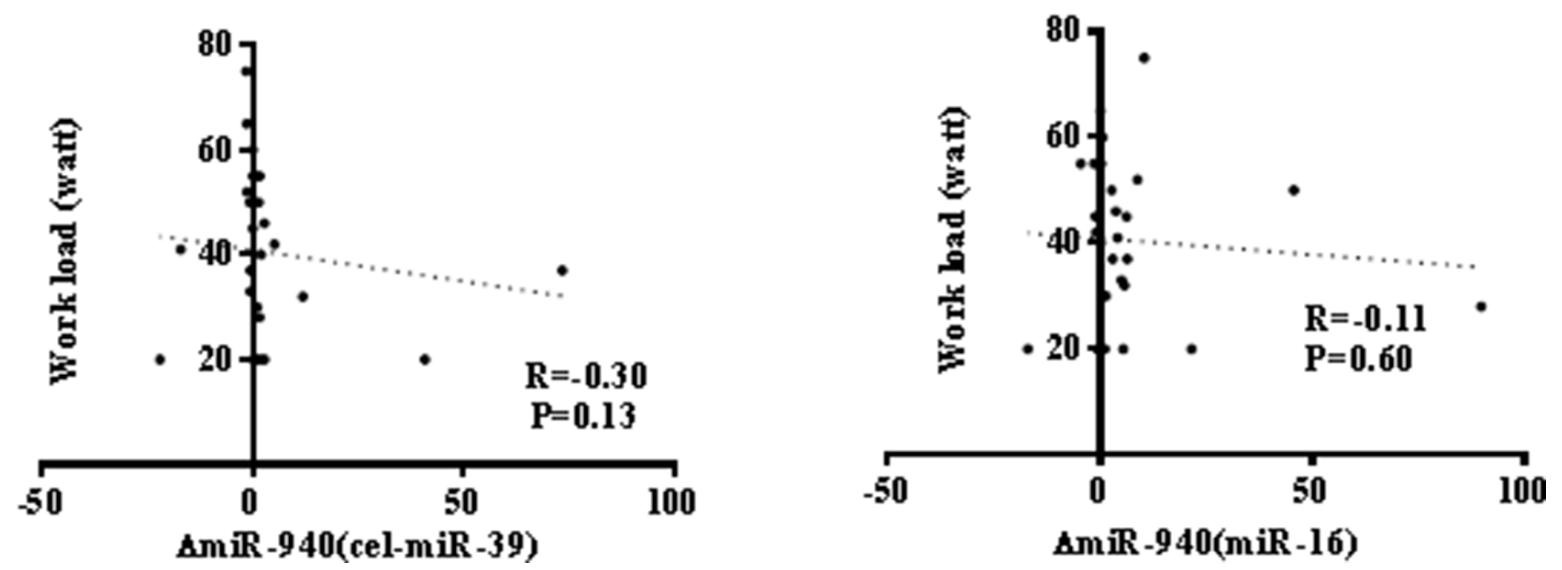

Figure 4: No correlation of changes of miR-21, miR-378 and miR-940 in exercise is observed with peak work load. (A) miR-21 (normalized to cel-miR-39); (B) miR-378 (normalized to cel-miR-39); (C) miR-940 (normalized to cel-miR-39); (D) miR-21 (normalized to miR-16); (E) miR-378 (normalized to miR-16); (F) miR-940 (normalized to miR-16). 
A

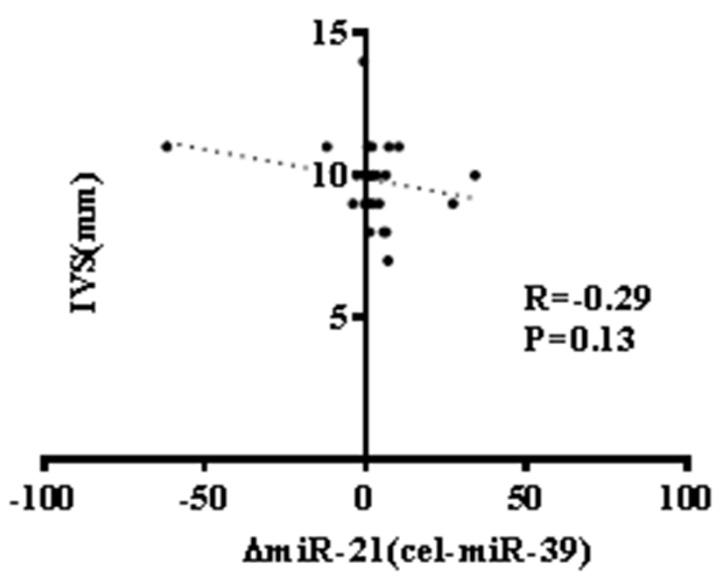

B

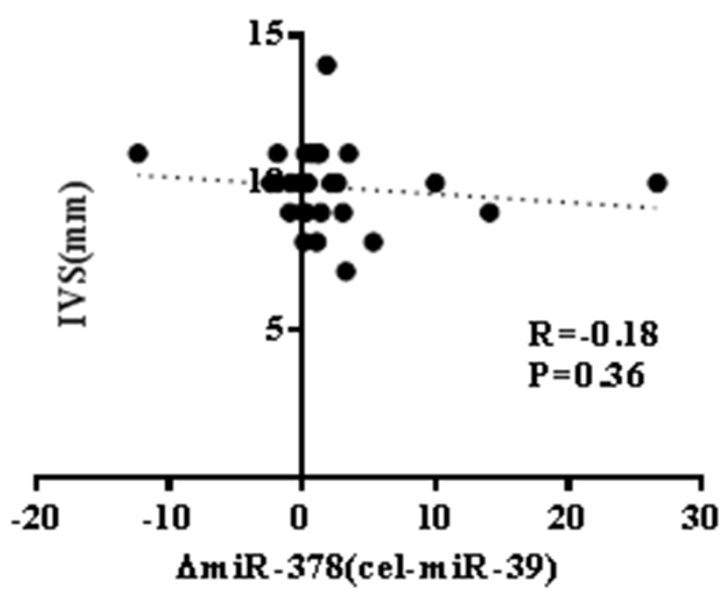

C

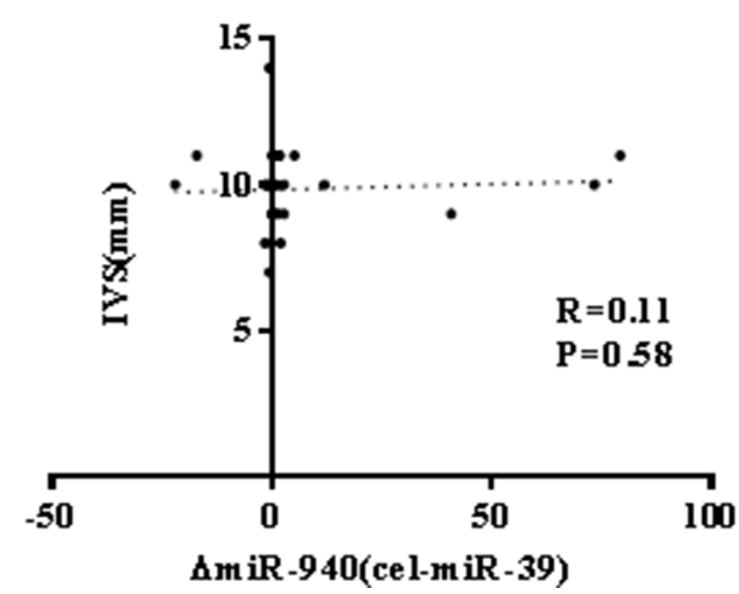

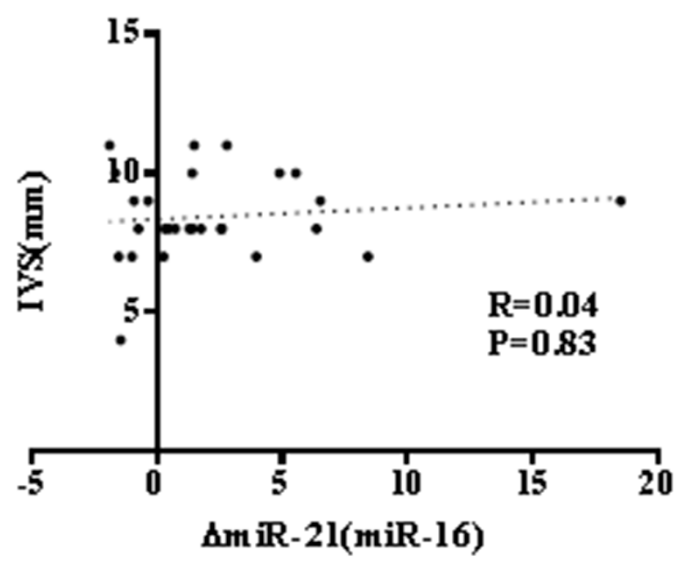

$\mathbf{E}$

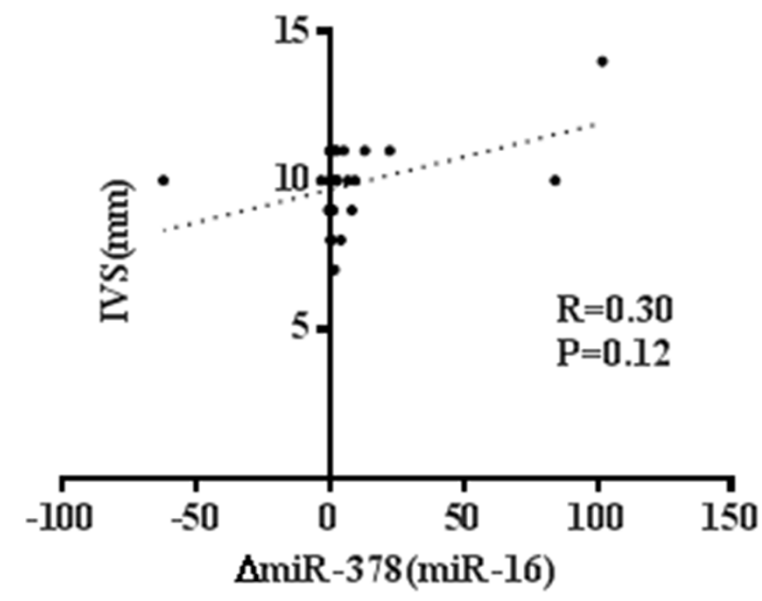

$\mathbf{F}$

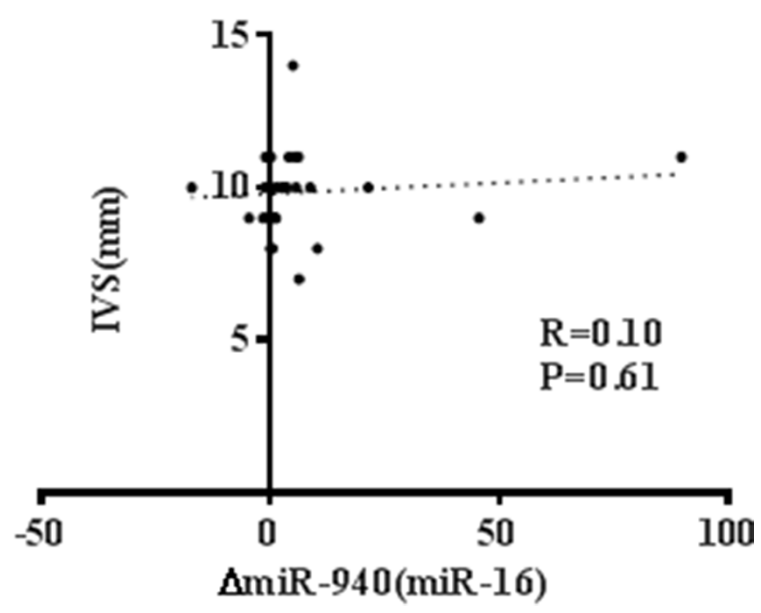

Figure 5: No correlation of changes of miR-21, miR-378 and miR-940 in exercise is observed with running speed at individual anaerobic lactate threshold $\left(\mathbf{V}_{\mathrm{IAS}}\right)$. (A) miR-21 (normalized to cel-miR-39); (B) miR-378 (normalized to cel-miR-39); (C) miR-940 (normalized to cel-miR-39); (D) miR-21 (normalized to miR-16); (E) miR-378 (normalized to miR-16); (F) miR-940 (normalized to miR-16). 
Table 4: Correlations between changes of microRNAs and biochemical indexes

\begin{tabular}{|c|c|c|c|c|c|c|c|c|c|c|c|c|c|}
\hline \multirow{2}{*}{\multicolumn{2}{|c|}{$\Delta$ miRNA }} & \multicolumn{2}{|c|}{ CK } & \multicolumn{2}{|c|}{ LDH } & \multicolumn{2}{|c|}{ СКМВ } & \multicolumn{2}{|c|}{ Troponin $\mathrm{T}$} & \multicolumn{2}{|c|}{ NT-ProBNP } & \multicolumn{2}{|c|}{ hs-CRP } \\
\hline & & $\mathbf{R}$ & $\mathbf{P}$ & $\mathbf{R}$ & $\mathbf{P}$ & $\mathbf{R}$ & $\mathbf{P}$ & $\mathbf{R}$ & $\mathbf{P}$ & $\mathbf{R}$ & $\mathbf{P}$ & $\mathbf{R}$ & $\mathbf{P}$ \\
\hline \multirow{2}{*}{ miR-21 } & cel-miR39 & 0.15 & NS & 0.39 & 0.04 & 0.06 & NS & -0.12 & NS & -0.33 & NS & 0.03 & NS \\
\hline & miR-16 & -0.40 & 0.04 & 0.20 & NS & 0.01 & NS & 0.06 & NS & 0.39 & 0.04 & 0.03 & $\mathrm{NS}$ \\
\hline \multirow{2}{*}{$\operatorname{miR}-378$} & cel-miR39 & 0.25 & NS & -0.46 & 0.02 & 0.32 & NS & -0.14 & NS & -0.29 & NS & 0.35 & NS \\
\hline & miR-16 & -0.3 & NS & 0.21 & NS & -0.11 & NS & -0.01 & NS & 0.33 & NS & -0.12 & NS \\
\hline \multirow{2}{*}{ miR-940 } & cel-miR39 & 0.41 & 0.03 & -0.21 & NS & 0.15 & NS & 0.06 & NS & -0.28 & NS & -0.06 & NS \\
\hline & miR-16 & -0.20 & NS & 0.34 & NS & 0.01 & NS & 0.22 & NS & 0.22 & NS & -0.22 & NS \\
\hline
\end{tabular}

miR, microRNA; NS, not significant.

As an initial "proof-of-concept" study, instead of using a more comprehensive but perhaps less reliable, highthroughput miRNA arrary technique, we used quantitative circulating miRNAs analysis checking selected miRNAs to decrease 'false positive hits'. The selected miRNAs have potential biological relevance in exercise including angiogenesis, inflammation, and ischemia adaptation. Among the miRNAs analyzed here are cardiac or muscle specific/enriched miRNAs including miR-1, miR-133a, miR-133b, miR-499, miR-208a, miR-208b, miR-378, miR-486, and miR-940. Among these, miR-940 is a miRNA recently found to be enriched in human cardiac tissue and potentially contribute to human Tetralogy of Fallot [29]. However, little information is known about this miRNA. Here we provide novel insights that this miRNA might participate in the response to exercise.

Several limitations of the present study should be highlighted. Firstly, a major weakness of the present study is that only one time point was selected for miRNAs determination after exercise. Considering circulating miRNA changes are likely to involve different mechanisms including cellular release or changed transcription, it would be interesting to compared their expression at the very acute phase (e.g. minutes after exercise) and at the prolonged phase (hours to days after exercise). Secondly, though beyond the scope the this study, the source and potential biological function of miR-21, miR-378, and miR-940 warrant to be clarified in the future. These miRNAs might be carried in the serum by Ago proteins or membrane vesicles like exosome [20, 30-32]. As cardiac or muscle specific miRNAs including miR-1, miR-133a, miR-133b, miR-499, miR-208a and miR-208b are not increased in this study, we speculated that the increase of miR-21, miR-378, and miR-940 in serum is an active release process, which might affect the cells nearby or in distant tissues and integrate the physiological adaptation to exercise as a whole organism. Thirdly, it would be interesting to see what genes are also effected by this change in miRNAs. Fourthly, to acquire a more complete picture of exercise-induced circulating miRNAs, a more complete characterization including clearance of miRNAs reported in this study and others in a larger number of human subjects is highly needed. Finally, the current study examines circulating miRNAs in response to an acute exhaustive exercise in CHF patients. Informed conclusions regarding the dynamic regulation of circulating miRNAs after sustained aerobic exercise training can not be extrapolated at this time and need to be further studied. Finally, whether there is a prognostic role for these miRNAs in long-term outcomes is also undetermined.

In conclusion, serum miR-21, miR-378, and miR-940 increase in response to acute exhaustive exercise in CHF patients. Future studies aim at defining the potential use of circulating miRNAs as biomarkers of exercise training and a better understanding of the direct biological function of circulating miRNAs in adaptation to exercise training are highly needed.

\section{MATERIALS AND METHODS}

\section{Participants}

Approval was obtained from the ethics committee of Shanghai Tongji Hospital. All human investigation complied with the principles outlined in the Declaration of Helsinki. All participants provided written informed consent before participating in the study. Between September 2013 and March 2014, twenty-eight patients with CHF underwent a symptom-limited incremental cardiopulmonary exercise test on a bicycle ergometer (GE, USA) using a standardized exercise 
protocol of revised Ramp10 programs at Shanghai Tongji Hospital as previously reported [26]. Briefly, the patient was recorded at rest on the treadmill for $3 \mathrm{~min}$. After that, the patient would cycle at $60 \mathrm{rpm}$ for another $3 \mathrm{~min}$ without any resistance. Then the work was increased at a pre-set rate (starting from $20 \mathrm{~J} / \mathrm{s}$ for $2 \mathrm{~min}$ and increased by $5 \mathrm{~J} / \mathrm{s}$ every $30 \mathrm{~s}$ ). The patient continued pedalling at $60 \mathrm{rpm}$ throughout the test, until they were exhausted. All participants did not have the experience of conducting cardiopulmonary exercise test at least for six months and were abstained from any physiological exercise for 1 day before and were conduct an overnight fasting. On the morning of testing, participants were permitted and encouraged to drink water (400-800 ml) but without caloric or electrolyte content.

\section{Serum sampling and RNA isolation}

Before and immediately after the cardiopulmonary exercise test, venous blood was collected in Serum Tubes (increased silica act clot activator, silicone-coated interior) and processed within 1 hour of collection. After a two-step centrifugation $\left(820 \times \mathrm{g}\right.$ for $10 \mathrm{~min}$ at $4^{\circ} \mathrm{C}$, and then $16000 \times \mathrm{g}$ for $10 \mathrm{~min}$ at $4^{\circ} \mathrm{C}$ ), the supernatant (serum) was transferred to RNase/DNase-free tubes and stored at $-80^{\circ} \mathrm{C}$ until further analysis.

The total RNA was isolated from the serum using a mirVana PARIS isolation kit (Ambion, Austin, Texas) according to the manufacturer's instructions for serum samples without enrichment for small RNAs. Briefly, $400 \mu \mathrm{L}$ of serum was used to extract the total RNA. Caenorhabditis elegans miR-39 (cel-miR-39) of 50 pmol/L was added as the spike-in control after the equal volume of denaturing solution was added. Each sample was eluted in $100 \mu \mathrm{L}$ of RNAse-free water.

\section{Determination of circulating miRNAs levels}

For quantitative miRNA analysis, Bulge-LoopTM miRNA qPCR Primer Sets (RiboBio) were used to detect selected miRNAs expressions by quantitative reverse transcription polymerase chain reactions (qRT-PCRs) with Takara SYBR Premix Ex Taq ${ }^{\mathrm{TM}}$ (TliRNaseH Plus) in the 7900HT Fast Real-Time PCR System as previously reported [26]. All qRT-PCR reactions were performed in triplicate, and the signal was collected at the end of every cycle. As there is no consensus on endogenous stable miRNAs in the circulation to act as house-keepers, the expression level of miRNAs in serum were normalized using spike-in cel-miR-39, which lacks sequence homology to human miRNAs. Besides that, miR-16, a representative miRNA enriched in serum, was also used in this study as an endogenous control.

\section{Biochemical measurements}

Creatine Kinase (CK), Creatine Kinase MB Isoenzyme (CK-MB), Troponin T (Tn-T), N-Terminal
Pro-Brain Natriuretic Peptide (NT-ProBNP) and high sensitive C Reactive Protein (hs-CRP) were determined by enzyme linked immunosorbent assays (ELISA) from Wuhan Xinqidi Biological Technology. Lactate dehydrogenase (LDH) was determined by ELISA kits from KeyGEN BioTECH.

\section{Statistical analysis}

Participants characteristics data were shown as mean $\pm \mathrm{SD}$. For the analysis of qRT-PCR data, the relative expression level for each miRNA was calculated using the $2^{-\Delta \Delta \mathrm{Ct}}$ method and the data were expressed as the mean $\pm \mathrm{SE}$. Paired samples were compared by Student's $t$ test or Wilcoxon's matched pairs test as appropriate for data distribution. Correlation analyses between changes of circulating miRNAs before and after an acute exercise and exercise capacity, muscle damage or inflammation were performed using the Spearman's or Pearson's method as appropriate for data distribution. $P$-values less than 0.05 were considered to be statistically significant.

\section{ACKNOWLEDGMENTS AND FUNDING}

This work was supported by the grants from National Natural Science Foundation of China (81570362 and 81200169 to J. Xiao; 81470515, 81270314 to J. Xu; 81400647 to Y. Bei; 81472158 to L Che; 81541007 to H. Wang), Innovation Program of Shanghai Municipal Education Commission (13YZ014 to J. Xiao), Foundation for University Young Teachers by Shanghai Municipal Education Commission (year 2012, to J. Xiao), Innovation fund from Shanghai University (sdcx2012038 to J. Xiao), Program for the integration of production, teaching and research for University Teachers supported by Shanghai Municipal Education Commission (year 2014, to J. Xiao) and Natural Science Foundation of Shanghai (14ZR1437900 to L. Che; 14ZR1438300 to H. Wang) and Shanghai Medical Guide Project from Shanghai Science and Technology Committee (134119a3000 to J. Xu; 14411971600 to J. Jiang)

\section{CONFLICTS OF INTEREST}

The authors declare there are no conflicts of interest.

\section{REFERENCES}

1. Ades PA, Keteyian SJ, Balady GJ, Houston-Miller N, Kitzman DW, Mancini DM, Rich MW. Cardiac rehabilitation exercise and self-care for chronic heart failure. JACC Heart Fail. 2013; 1:540-547.

2. Loffredo FS, Nikolova AP, Pancoast JR, Lee RT. Heart failure with preserved ejection fraction: Molecular pathways of the aging myocardium. Circ Res. 2014; 115:97-107.

3. Duncker DJ, van Deel ED, de Waard MC, de Boer M, Merkus D, van der Velden J. Exercise training in adverse cardiac remodeling. Pflugers Arch. 2014; 466:1079-1091. 
4. Adams V, Schuler G. Heart failure: Exercise training-a magic bullet for chronic heart failure? Nat Rev Cardiol. 2012; 9:677-678.

5. O'Connor C. It is time to exercise change for heart failure. JACC Heart Fail. 2013; 1:549-550.

6. Gargiulo P, Olla S, Boiti C, Contini M, Perrone-Filardi P, Agostoni P. Predicted values of exercise capacity in heart failure: where we are, where to go. Heart Fail Rev. 2014; 19:645-653.

7. Fernandes T, Soci UP, Oliveira EM. Eccentric and concentric cardiac hypertrophy induced by exercise training: Micrornas and molecular determinants. Braz J Med Biol Res. 2011; 44:836-847.

8. Liang C, Yu XJ, Guo XZ, Sun MH, Wang Z, Song Y, Ni QX, Li HY, Mukaida N, Li YY. MicroRNA-33a-mediated downregulation of Pim-3 kinase expression renders human pancreatic cancer cells sensitivity to gemcitabine. Oncotarget. 2015; 6:14440-14455. doi: 10.18632/oncotarget.2929.

9. Wang BW, Wu GJ, Cheng WP, Shyu KG. Microrna-208a increases myocardial fibrosis via endoglin in volume overloading heart. PLoS One. 2014; 9:e84188.

10. Amodio N, Rossi M, Raimondi L, Pitari MR, Botta C, Tagliaferri P, Tassone P. miR-29s: a family of epi-miRNAs with therapeutic implications in hematologic malignancies. Oncotarget. 2015; 6:12837-12861. doi: 10.18632/ oncotarget. 3805 .

11. Fisher JN, Terao M, Fratelli M, Kurosaki M, Paroni G, Zanetti A, Gianni M, Bolis M, Lupi M, Tsykin A, Goodall GJ, Garattini E. MicroRNA networks regulated by all-trans retinoic acid and Lapatinib control the growth, survival and motility of breast cancer cells. Oncotarget. 2015; 6:13176-13200. doi: 10.18632/oncotarget.3759.

12. Chun-Zhi Z, Lei H, An-Ling Z, Yan-Chao F, Xiao Y, Guang-Xiu W, Zhi-Fan J, Pei-Yu P, Qing-Yu Z, Chun-Sheng K. MicroRNA-221 and microRNA-222 regulate gastric carcinoma cell proliferation and radioresistance by targeting PTEN. BMC Cancer. 2010; 10:367.

13. Smith AR, Marquez RT, Tsao WC, Pathak S, Roy A, Ping J, Wilkerson B, Lan L, Meng W, Neufeld KL, Sun XF, Xu L. Tumor suppressive microRNA-137 negatively regulates Musashi-1 and colorectal cancer progression. Oncotarget. 2015; 6:12558-12573. doi: 10.18632/oncotarget.3726.

14. Ahmad A, Ginnebaugh KR, Sethi S, Chen W, Ali R, Mittal S, Sarkar FH. miR-20b is up-regulated in brain metastases from primary breast cancers. Oncotarget. 2015; 6:12188-12195. doi: 10.18632/oncotarget.3664.

15. Jiang J, Zhang Y, Guo Y, Yu C, Chen M, Li Z, Tian S, Sun C. MicroRNA-3127 promotes cell proliferation and tumorigenicity in hepatocellular carcinoma by disrupting of PI3K/AKT negative regulation. Oncotarget. 2015; 6:6359-6372. doi: 10.18632/oncotarget.3438.

16. Pescador N, Pérez-Barba M, Ibarra JM, Corbatón A, Martínez-Larrad MT, Serrano-Ríos M. Serum circulating
microRNA profiling for identification of potential type 2 diabetes and obesity biomarkers. PLoS One. 2013; 8:e77251.

17. Ai J, Zhang R, Li Y, Pu J, Lu Y, Jiao J, Li K, Yu B, Li Z, Wang $\mathrm{R}$, Wang $\mathrm{L}$, Li Q, Wang $\mathrm{N}$, et al. Circulating microRNA-1 as a potential novel biomarker for acute myocardial infarction. Biochem Biophys Res Commun. 2010; 391:73-77.

18. Turchinovich A, Weiz L, Langheinz A, Burwinkel B. Characterization of extracellular circulating microrna. Nucleic Acids Res. 2011; 39:7223-7233.

19. Achberger S, Aldrich W, Tubbs R, Crabb JW, Singh AD, Triozzi PL. Circulating immune cell and microRNA in patients with uveal melanoma developing metastatic disease. Mol Immunol. 2014; 58:182-186.

20. Arroyo JD, Chevillet JR, Kroh EM, Ruf IK, Pritchard CC, Gibson DF, Mitchell PS, Bennett CF, Pogosova-Agadjanyan EL, Stirewalt DL, Tait JF, Tewari M. Argonaute2 complexes carry a population of circulating microRNAs independent of vesicles in human plasma. Proc Natl Acad Sci U S A. 2011; 108:5003-5008.

21. Sawada S, Kon M, Wada S, Ushida T, Suzuki K, Akimoto T. Profiling of circulating microRNAs after a bout of acute resistance exercise in humans. PLoS One. 2013; 8:e70823.

22. Aoi W, Ichikawa H, Mune K, Tanimura Y, Mizushima K, Naito Y, Yoshikawa T. Muscle-enriched microRNA miR-486 decreases in circulation in response to exercise in young men. Front Physiol. 2013; 4:80.

23. Baggish AL, Park J, Min PK, Isaacs S, Parker BA, Thompson PD, Troyanos C, D'Hemecourt P, Dyer S, Thiel M, Hale A, Chan SY. Rapid upregulation and clearance of distinct circulating microRNAs after prolonged aerobic exercise. J Appl Physiol. 2014; 116:522-531.

24. Gomes CP, Oliveira-Jr GP, Madrid B, Almeida JA, Franco OL, Pereira RW. Circulating miR-1, miR-133a, and miR-206 levels are increased after a half-marathon run. Biomarkers : biochemical indicators of exposure, response, and susceptibility to chemicals. 2014; 19:585-589.

25. Nielsen S, Åkerström T, Rinnov A, Yfanti C, Scheele C, Pedersen BK, Laye MJ. The miRNA plasma signature in response to acute aerobic exercise and endurance training. PloS One. 2014; 9:e87308.

26. Liu X, Xiao J, Zhu H, Wei X, Platt C, Damilano F, Xiao C, Bezzerides V, Boström P, Che L, Zhang C, Spiegelman BM, Rosenzweig A. miR-222 is necessary for exercise-induced cardiac growth and protects against pathological cardiac remodeling. Cell Metab. 2015; 21:584-595.

27. Mooren FC, Viereck J, Kruger K, Thum T Circulating micrornas as potential biomarkers of aerobic exercise capacity. Am J Physiol Heart Circ Physiol. 2014; 306:H557-563.

28. Baggish AL, Hale A, Weiner RB, Lewis GD, Systrom D, Wang F, Wang TJ, Chan SY. Dynamic regulation of circulating microRNA during acute exhaustive exercise and sustained aerobic exercise training. J Physiol. 2011; 589:3983-3994. 
29. Liang D, Xu X, Deng F, Feng J, Zhang H, Liu Y, Zhang Y, Pan L, Liu Y, Zhang D, Li J, Liang X, Sun Y, et al. miRNA-940 reduction contributes to human Tetralogy of Fallot development. J Cell Mol Med. 2014; 18:1830-1839.

30. Lasser C. Exosomal rna as biomarkers and the therapeutic potential of exosome vectors. Expert Opin Biol Ther. 2012; 12: S189-197.

31. Mobergslien A, Sioud M. Exosome-derived mirnas and cellular mirnas activate innate immunity. J Innate Immun. 2014; 6:105-110.
32. Bang C, Batkai S, Dangwal S, Gupta SK, Foinquinos A, Holzmann A, Just A, Remke J, Zimmer K, Zeug A, Ponimaskin E, Schmiedl A, Yin X, et al. Cardiac fibroblastderived microRNA passenger strand-enriched exosomes mediate cardiomyocyte hypertrophy. Clin Invest. 2014; 124:2136-2146. 